\title{
Differentiation of Myeloid Cell Lines Correlates with a Selective Expression of RIZ Protein
}

\author{
Patrizia Gazzerro' ${ }^{1}$, Paola Bontempo ${ }^{1}$, Ettore M. Schiavone ${ }^{2}$, Ciro Abbondanza ${ }^{1}$, Bruno Moncharmont ${ }^{1}$, \\ Ignazio Armetta ${ }^{1}$, Nicola Medici ${ }^{3}$, Mariacarla De Simone ${ }^{2}$, Ernesto Nola ${ }^{1}$, Giovanni A. Puca ${ }^{1}$, and \\ Anna Maria Molinari ${ }^{1}$ \\ ${ }^{1}$ Istituto di Patologia generale ed Oncologia, Seconda Università degli studi di Napoli, Naples, Italy \\ 2 XIX Divisione di Ematologia, Azienda ospedaliera “A. Cardarelli," Naples, Italy \\ ${ }^{3}$ Istituto pluridisciplinare di Patologia generale, Università degli studi di Messina, Messina, Italy \\ Accepted May 15, 2001
}

\begin{abstract}
Background: The retinoblastoma-interacting zinc-finger gene RIZ is expressed in two forms (RIZ1 and RIZ2) that differ for the presence near the N-terminus of RIZl of a conserved domain, defined PR (PRDI-BFI-RIZ homology), homologous to a similar domain present in other proteins recognized as tumor suppressor gene products. The RIZl form is usually absent or expressed at low levels in tumor cells, whereas RIZ2 is frequently expressed. We investigated a possible involvement of RIZl in differentiation control using a myeloid cell maturation model that is easily modulated by retinoids and other agents.

Materials and Methods: HL60 or NB4 cell lines or patients' leukemic promyelocytes were treated with alltrans-retinoic acid or other agents to induce differentiation. RIZ gene expression was determined with reverse transcriptase polymerase chain reaction (RT-PCR) and RNase protection assay. Immunocytochemistry was performed to assess variation of the intracellular distribution of RIZ protein on all-trans-retinoic acid treatment. Forced expression of RIZl protein was obtained with a recombinant adenovirus containing RIZI cDNA.
\end{abstract}

Results: Treatment with retinoic acid induced a selective expression of RIZl in HL60 cell line. Retinoic acid effect was maximal at 7 days and correlated to the granulocytic differentiation of cells. A similar effect was obtained in retinoic acid-sensitive NB4 cell line or in patients' leukemic promyelocytes, but not in the retinoic acidresistant cell line NB4.007/6 or in the U937 cell line. Selective expression of RIZl was also induced by 12-Otetradecanoyl-phorbol-13-acetate in the U937 and HL60 cell lines and by 1,25-dihydroxyvitamin $\mathrm{D}_{3}$ only in HL60 cells. In HL60 cells, RIZl was also induced by activation of a retinoid $\alpha$ receptor-independent maturation pathway based on retinoid $X$ receptor agonist and protein kinase $A$ synergism. In addition, retinoic acid produced a redistribution of the antigen within the nucleus in these cells. Forced expression of RIZl protein induced growth arrest and death of HL60 cells.

Conclusions: The correlation between the selective expression of RIZ1 induced by retinoic acid, 12-O-tetradecanoylphorbol-13-acetate, or 1,25-dihydroxyvitamin $\mathrm{D}_{3}$ and differentiation suggested that RIZ protein was involved in myeloid cell differentiation induced by these agents.

\section{Introduction}

The retinoblastoma-interacting zinc-finger protein RIZ is a 1719-aa protein, containing eight zinc finger motifs, that has been identified for its ability to bind the retinoblastoma gene product (1). RIZ is mainly expressed in neuroendocrine tissues, with a ubiquitous low level of expression. RIZ protein is able to modulate gene expression, either positively or negatively, by direct interaction with DNA as a transcription factor, as well as a co-activator interacting with other trans-acting protein moieties. RIZ gene product

Address correspondence and reprint requests to: Bruno Moncharmont, MD, Istituto di Patologia generale ed Oncologia, Facoltà di Medicina e Chirurgia, Seconda Università degli studi di Napoli, Larghetto Sant'Aniello a Caponapoli, 2, I-80138 Naples, Italy. Phone: +39081 5665675; fax: +39081 5665695; e-mail: bruno.moncharmont@unina2.it

P. G. and P. B. equally contributed to the work. is a weak activator of heme-oxygenase gene and a repressor of herpes simplex virus (HSV) thymidine kinase promoter. These opposite effects are mediated by RIZ protein binding to different cis-acting elements, such as the macrophage-specific 12-O-tetradecanoylphorbol-13-acetate (TPA)-responsive element (MTE) in the human heme-oxygenase promoter (2) or the Sp-1 element in the HSV thymidine kinase promoter (3). In addition, it could also act as regulator of the GATA family of transcription factors (4). A more provocative hypothesis on the function of RIZ gene product is suggested by its interaction with the $R b$ protein (1). In this way RIZ protein could be involved in the control of cell proliferation. The Rb-binding domain of RIZ is contained in an acidic region at the $\mathrm{N}$-terminus, sharing structural and antigenic similarities with the ElA viral oncogene, and it binds to the same domain of $\mathrm{Rb}$, suggesting that it could be the endogenous equivalent of the viral protein (1). 
The main interest raised by RIZ gene is related to its role as a putative tumor suppressor. It has been physically mapped to chromosome $1 \mathrm{p} 36$, a region deleted in many human cancer cell lines as well as in solid tumors $(2,5)$. Furthermore, loss of heterozygosity at 1 p36 has been observed in human breast cancer and, very recently, frameshift mutations of the RIZ gene have been detected in colorectal cancer (6). A distinctive feature of RIZ protein is the presence of a PR domain (PRDI-BFl-RIZ homology) near the N-terminus. This domain is structurally related to a similar domain present in other gene products functioning as negative regulators of growth and characterizes a subfamily of Krüppel-like zincfinger genes (7). PR domain-containing proteins of this family are expressed and present in the nucleus of quiescent or differentiated cells, whereas they are absent in the nucleus of proliferating or neoplastic cells. MDS 1 and EVII are two separate genes producing a fusion transcript in normal cells. The PR domain thus produced by intergenic splicing is disrupted in leukemic cells by chromosome translocation (8). Another PR domain-containing gene product, the transcription factor PRDI-BFl/BLIMP1, is induced during normal B-cell differentiation. Its forced expression in B-cell lymphoma cell lines induces B-cell differentiation (9) that is blocked by a dominant-negative mutant lacking the PR domain (10). SC-1 gene product, another member of the family, is associated with the NGF receptor and translocated to the nucleus upon ligand stimulation or serum starvation of COS cells (11). In the RIZ gene, the PR domain is selectively expressed in one of two transcripts. Only the longer gene product (RIZ1) contains the PR domain; the shorter one (RIZ2) is derived from a transcript initiating at the end of exon 6 and directed by an internal promoter (7). More than one transcript, with a differential expression of the PR domain, is also produced by a new member of the family, the PFMl gene (12). The PR-plus product RIZl is commonly underexpressed in cancer cells, where, in contrast, the RIZ2 transcript is detected. Furthermore, forced RIZl expression in cultured cancer cells induces growth arrest or apoptosis $(13,14)$.

Recently, we suggested a role of RIZ protein as effector of the mitogenic response of estradiol. In MCF-7 cells, where estradiol is an inducer of cell proliferation, estrogen receptor is associated with RIZ protein in a hormone-dependent manner. Furthermore, estradiol treatment produces a redistribution of RIZ protein within the cell (15). We planned to investigate whether RIZ protein was also involved in differentiation control, using a welldefined model of myeloid cell maturation. In myeloid leukemias, neoplastic cells do not follow the normal developmental sequence leading to arrest of proliferation after the myelocyte stage, differentiation and, eventually, death by apoptosis. They remain instead at an intermediate stage of differentiation, indefinitely capable of cell division. In addition, apoptotic programs are usually suppressed. Most cases of acute promyelocytic leukemia show the $15 ; 17$ translocation, juxtaposing the retinoic acid receptor- $\alpha$ gene to the PML gene and yielding in a fusion gene product $(16,17)$. All-trans-retinoic acid (ATRA) induces maturation of several promyelocytic cell lines. In addition, patient treatment with this agent results in the differentiation of leukemic promyelocytes, inducing a remission of the disease $(18,19)$. In this report we show that treatment of myeloid cell lines, or patients' leukemic promyelocytes, with agents promoting their differentiation induces a selective expression of the tumor-suppressor form of RIZ gene product and modified its immunocytochemical detection properties.

\section{Materials and Methods} Cell Culture

HL60, NB4, NB4.007/6 (20) or U937 cells were grown in RPMI 1640 supplemented with $10 \%$ fetal calf serum (FCS) (heat inactivated), $2 \mathrm{mM}$ of L-glutamine, $100 \mathrm{U} / \mathrm{ml}$ of penicillin, $100 \mu \mathrm{g} / \mathrm{ml}$ of streptomycin, and gentamicin $50 \mu \mathrm{g} / \mathrm{ml}$ in a $5 \% \mathrm{CO}_{2}$ environment. Promyelocytic blasts were obtained from patients affected with acute promyelocytic leukemia (APL) before in vivo treatment with ATRA or chemotherapy. Diagnosis of APL was established by morphology, according to French-American-British group criteria, and confirmed by cytogenetic and molecular detection of PML/RAR $\alpha$ transcripts. All patients responded to ATRA therapy. Mononuclear cells were isolated from peripheral blood by isopyknic sedimentation through Ficoll-Hypaque $(d=1.077$; Seromed, Biochem KG, Roskilde, Denmark) at $300 \times g$ for $20 \mathrm{~min}$, followed by two washes in RPMI 1640 . Isolated cells were grown for $24 \mathrm{hr}$ in medium supplemented with $20 \%$ FCS and then incubated in the indicated conditions. Infection of HL60 cells with the adenovirus vectors AdRIZl and AdGFP was performed as described (13).

\section{Reverse Transcriptase Polymerase Chain Reaction}

Total RNA was extracted from human cell lines by guanidinum thiocyanate-isopropanol method. Reverse transcription (RT) was performed using Moloney murine leukemia virus reverse transcriptase and random oligonucleotide primer. The first strand cDNA was then amplified using two different sets of primers. The forward primer Riz-118F (5'-CTGGATCCACCCGGATTGGTGTCTGGG-3') and the antisense primer, Riz-438R (5'-TCGGATCCAGGGTTGTCTTCCCCATTGTACC-3') were used to amplify a fragment exclusive of RIZl. The forward primer Riz-649F (5'-CTGGATCCTCAGCCTCAGCACTTGAGCAG-3') and the antisense primer, Riz-975R (5'-TCGGATCCTGTTTTTGGTTCCTCTAATAAATCTTC-3') were used to amplify a fragment common to both RIZ1 and RIZ2. The primers for amplification of human alpha actinin were 
AlF (5'-ATGATCTGGACCATCATCCT-3') and AIR (5'-TTCCAGCTGRTGTGGAGGTCC-3'). Polymerase chain reactions (PCR) were performed $25 \mathrm{sec}$ at $93{ }^{\circ} \mathrm{C}$, $1 \mathrm{~min}$ at $62^{\circ} \mathrm{C}$ (or at $63^{\circ} \mathrm{C}$ with Riz- $118 \mathrm{~F}$ and Riz$438 \mathrm{R}$ primers), and $1 \mathrm{~min}$ at $69 \cdot \mathrm{C}$ for $25-28$ cycles. Amplified DNA was extracted with chloroform and electrophoresed in a $2 \%$ agarose-gel in $0.5 \times$ TBE.

\section{RNase Protection Assay}

For generation of the sequence-specific hybridization antisense probe, an amplified fragment from MCF-7-cell total RNA with RT-PCR using Riz-564R (5' - TCTGGCTCACTTGTCTTCAGTTGT -3') and Riz$118 \mathrm{~F}$ primers was cloned downstream of the bacteriophage promoter ( $E c o R V$ site) into a pBluescriptSK vector (pBS-SK-564R/118F). The cloned insert was controlled by direct sequencing and the plasmid linearized with ApoI. Labeled antisense RNA probe was produced by T7 RNA polymerase transcription in the presence of $\alpha\left[{ }^{32} \mathrm{P}\right]$ UTP and gel purified. RNase protection assay was performed with the RPA II ${ }^{\mathrm{TM}}$ kit (Ambion Inc., Austin, TX, USA). Protected products were analyzed on a DNA sequencing gel followed by autoradiography.

\section{RIZ Protein Detection}

$\left[{ }^{35}\right.$ S]met-labeled RIZ protein was produced by transcription of NotI-linearized pCT-RIZ (21) with T7 RNA polymerase. RNA was phenol extracted, ethanol precipitated, and resuspended in water. A $0.5-\mu \mathrm{g}$ aliquot of RNA was transcribed with reticulocyte lysate (Promega, Madison, WI, USA) according to manufacturer's instruction. Immunoprecipitation with mouse monoclonal antibody RZ1723 to RIZ protein was performed as described (21).

The transfection vector pEGFP-Cl (Clontech Laboratories Inc., Palo Alto, CA, USA) was digested with BamHI and ligated with PCR-amplified fragments of RIZ cDNA coding for the proline-rich domain (aa 951-1052) or for the CR2 (E1Alike) domain (aa 217-325) with BamHI cohesive ends, to obtain the pEGFP-RIZ(951-1052) and pEGFP-RIZ(217-325) plasmids, respectively. The correct orientation of the insert fused with the green fluorescent protein (GFP) was verified by restriction analysis. Permanently transfected MCF-7 cells were obtained by electroporation $(230 \mathrm{~V}, 960 \mu \mathrm{F})$ of $2 \times 10^{6}$ cells in the presence of $10 \mu \mathrm{g}$ of plasmid DNA, followed by selection in the presence of $0.8 \mu \mathrm{g} / \mathrm{ml}$ of $\mathrm{G} 418$.

Preparations of the total cellular extract (22) or of the nuclear extract $(15,23)$ were performed as described. Electrophoresis and Western blot analysis were performed, as described elsewhere (15), with mouse monoclonal antibody RZ1723 to RIZ protein (21) or with rabbit affinity-purified polyclonal antibodies SC-317 to p130 (dilution 1:7000, Santa Cruz Biotechnology Inc., Santa Cruz, CA, USA), or with polyclonal antibodies to GFP (dilution 1:200, Clontech Laboratories Inc.).

\section{Morphology and Immunocytochemistry}

Cells grown for 7 days in the presence of $1 \mu \mathrm{M}$ ATRA or vehicle alone were collected on slides with a Citospin centrifuge. Cell morphology was analyzed after May-Grünwald staining. For immunocytochemistry, cells were fixed with $4 \%(\mathrm{w} / \mathrm{v})$ formaldehyde in phosphate-buffered saline (PBS) for $10 \mathrm{~min}$, and permeabilized with $0.2 \%$ Triton X-100 in PBS for 10 min (where indicated), then treated with $0.1 \%$ hydrogen peroxide in methanol for $15 \mathrm{~min}$ at $4^{\circ} \mathrm{C}$, air-dried, and blocked with PBS containing $2 \%$ normal goat serum and $5 \%$ bovine serum albumin (BSA). After three washes with PBS, sections were incubated overnight at $4^{\circ} \mathrm{C}$ with RZ1723 hybridoma cell supernatant (21), or antibodies to proliferating cell nuclear antigen (PCNA) (diluted 1:200; Sigma, St. Louis, MO, USA) or control antibodies. After three washes with PBS, sections were incubated with biotinylated goat anti-mouse IgG (Boehringer, Mannheim, Germany) for $1 \mathrm{hr}$ at room temperature and further processed with ABC kit (Vector Laboratories, Burlingame, CA, USA) following the manufacturer's instruction. Slides were washed in water, dehydrated with ethanol and xylene, and then mounted with Eukitt mounting medium (O. Kindler GmbH, Freiburg, Germany). For vital staining, cells were mixed with a freshly diluted $0.01 \%$ acridine orange solution in potassium phosphate buffer ( $\mathrm{pH}$ 6.0).

\section{Flow Cytometry}

Expression of CD11b and CD14 was analyzed by direct immunofluorescence. Cells were washed with PBS and labeled with the specific fluoresceine isothiocyanate-labeled (FITC) mouse monoclonal antibody (Becton Dickinson, Mountain View, CA, USA). Cells were washed twice with PBS, fixed in $1 \%$ paraformaldehyde solution in PBS, and analyzed using a FACScalibur instrument (Becton Dickinson).

\section{Results}

HL60 cells were induced to differentiate by treatment with ATRA (24). Expression of RIZ gene was assayed by RT-PCR of total cellular RNA, using two alternative sets of primers (Fig. 1A). The first set amplified a sequence contained in the $5^{\prime}$ end of the transcript and, therefore, coding for an amino acid sequence exclusive of RIZl protein (aa 40-146). The second set amplified a sequence downstream the transcription initiation of RIZ2 protein and that was translated in both forms of RIZ gene product (aa 216-325). The amplified bands were, therefore, representative of the amount of both RIZ1 and RIZ2 transcripts or exclusively of RIZ1. ATRA treatment induced an increase of RIZl transcript after 3 or more days (Fig. 1B, lanes $a-f)$. A similar effect was obtained with the 9-cis-retinoic acid (data not presented). The limited parallel increase of total RIZ mRNA suggested that the RIZ2 expression was unaffected or rather suppressed by the treatment. Induction of granulocyte 
A

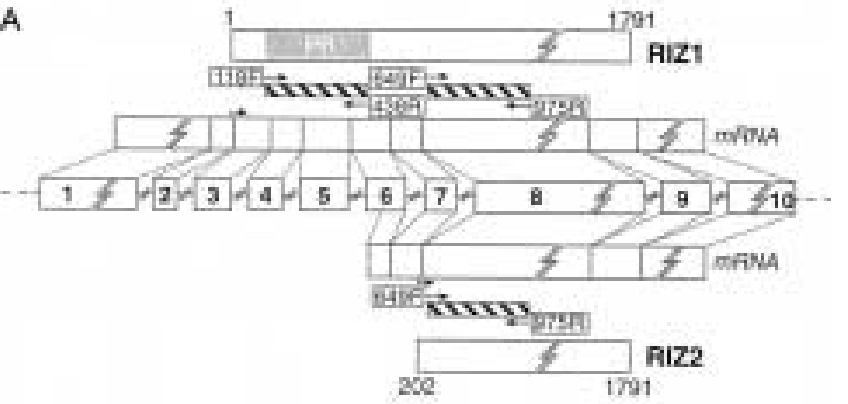

B

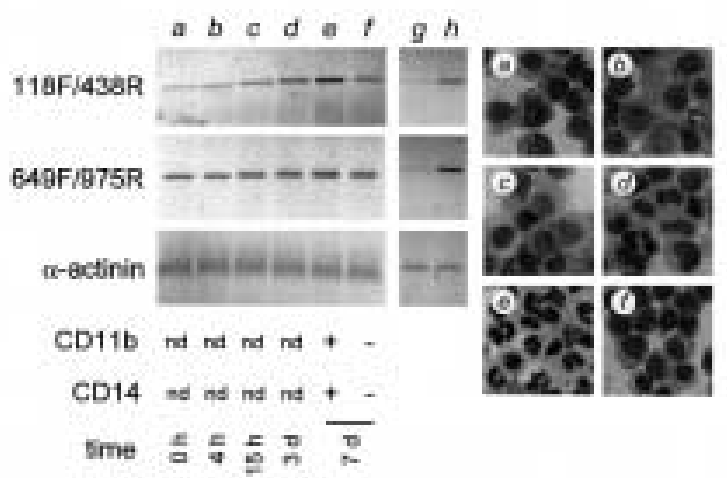

C

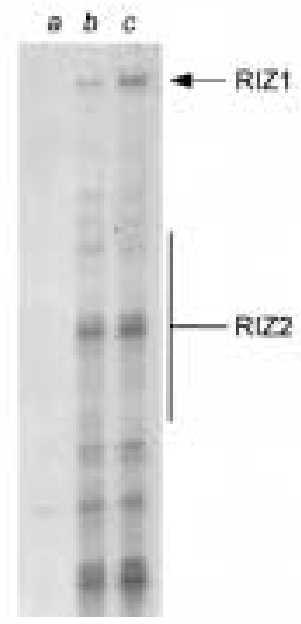

Fig. 1. Selective expression of RIZ1 induced by ATRA in HL60 cell line correlated with differentiation. (A) schematic diagram of the 5' end of RIZ gene, RIZl and two transcripts and proteins. Hatched bars represent the fragments amplified with the indicated sets of primers. (B) Electrophoretic analysis of the fragments obtained by reverse transcriptase-polymerase chain reaction (RT-PCR) of mRNA extracted from untreated HL60 cells $(a, f$ and $g$ ), treated with $1 \mu \mathrm{M}$ ATRA for $4 \mathrm{hr}(b), 15 \mathrm{hr}(c), 3$ days (d), 7 days (e), or with $1 \mu \mathrm{M}$ SR 11237 and $100 \mu \mathrm{M}$ 8CTP-cAMP $(h)$. The picture shows the negative image of the gel stained with ethidium bromide; on the left side is reported the set of primers used for amplification. Below is reported the expression of the differentiation markers CDI1b and CD14 determined by flow cytometry. On the right side, micrographs of May-Grünwald staining of the cells.

(C) RNase protection analysis of $10 \mu \mathrm{g}$ tRNA (a), or of $10 \mu \mathrm{g}$ total mRNA extracted from untreated HL60 cells $(b)$ or treated with $1 \mu$ M ATRA for 7 days (c). Arrows on the left side indi- differentiation of HL60 cell was also obtained through an alternative pathway based on the crosstalk between retinoid X receptor (RXR) and protein kinase A (25). Cell treatment with the RXR ligand SR 11237 and with the cell-permeable protein kinase A-agonist 8CTP-cAMP produced, after 4 days, a similar induction of RIZl selective expression (Fig. 1B, lane h). Granulocyte differentiation induced by ATRA was monitored by morphologic analysis or flow cytometry assay of CDI1b and CDI4. Both methods indicated a time correlation between ATRA-induced differentiation and RIZl expression. The selective increase of RIZ1 mRNA induced by ATRA treatment was confirmed by RNase protection assay (Fig. 1C) with an antisense RIZ probe covering the region between aa 111 and 196 of human RIZ cDNA. The longest fragment (254 nt) represented RIZl and it is markedly more abundant in the ATRA-treated cells. In Figure lc, the expected migration of RIZ2-protected fragments is also indicated.

The monoclonal antibody RZ1723 was used to assay RIZ protein in the nuclear extracts. This monoclonal antibody, obtained from a mouse immunized with a recombinant fragment of RIZ protein, was able to immunoprecipitate the full-length in vitro synthesized antigen (Fig. 2A). Furthermore, it was able to selectively immunodetect, by Western blot analysis of the total cell extract, the fragment of RIZ protein containing the epitope (aa 217-325), contained in a GFP recombinant protein expressed by permanently transfected COS cells. Western blot analysis with the antibody RZ1723 of the nuclear extract of HL60 cells treated for 7 days with ATRA showed an increase of RIZ protein signal, thus indicating a corresponding increase of the protein following gene transcription. The 220-kDa band contained both RIZ1 and RIZ2 protein, because the $8 \%$ acrylamide gel used in the experiment did not resolve the two forms and the antibody was unable to discriminate between them. Bands of smaller molecular weight were specifically visualized by the antibody in the extract from ATRA-treated cell, suggesting the concurrent formation of degradation products. Western blot analysis of the same nuclear extracts with antibodies to $\mathrm{Rb}$ protein or to p130 (SC-317) indicated that ATRA was not modifying the expression of the former (data not shown), whereas it dramatically increased the expression of the latter (Fig. 2C). This was consistent with the described role of p130 in granulocytic differentiation (26).

Another ATRA-responsive promyelocytic cell line, NB4, showed by RT-PCR analysis the same selective induction of RIZl after 7-day treatment with retinoic acid, whereas the ATRA-unresponsive variant NB4.007/6 (NB4r) (20) did not (Fig. 3A).

cate the migration of RIZ1 or RIZ2 protected fragments calculated from mouse actinin standards migration. 
A

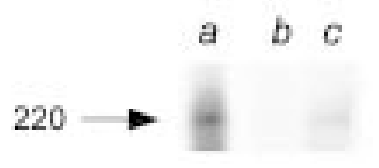

B

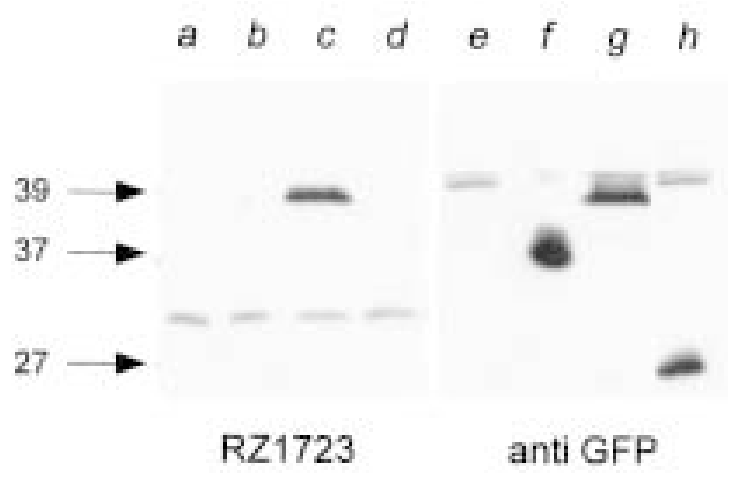

C

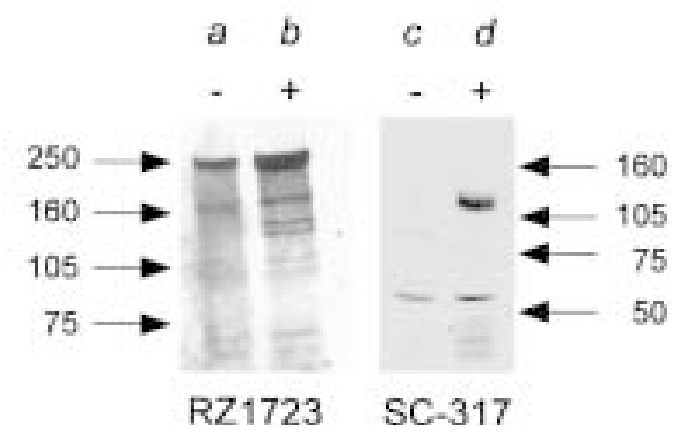

Fig. 2. Expression of RIZ protein detected with the monoclonal antibody RZ1723. (A) Autoradiograph of the $\left[{ }^{35} S\right]$ metlabeled full-length RIZ protein synthetized in vitro in reticulocyte lysate (lane $a$ ), immunoprecipitated with the monoclonal antibody RZ1723 (lane $c$ ), or by mouse control antibodies (lane $b$ ). Arrow on the sides indicates the molecular weight of the band calculated from migration of standard proteins (kDa). (B) Western blot analysis with the antibody RZ1723 to RIZ protein or with antibodies to GFP of total cell extracts from COS cells mock transfected (lanes $a$ and $e$ ) or transfected with pEGFP-RIZ(951-1052) (lanes $b$ and $f$ ), pEGFP-RIZ(217-325) (lanes $c$ and $g$ ), or pEGFP-Cl (lanes $d$ and $h$ ). Arrows on the sides indicate the migration of standard proteins (kDa).

(C) Western blot analysis of nuclear extract of HL60 cells untreated $(-)$ or treated with $1 \mu \mathrm{M}$ of ATRA for 7 days $(+)$ with the antibody RZ1723 to RIZ protein (lanes $a$ and $b$ ) or SC-317 to p130 (lanes $c$ and $d$ ), as indicated below. Arrows on the sides indicate the migration of standard proteins $(\mathrm{kDa})$.

Leukemic promyelocytes from two different APL patients, whose disease was controlled by therapy with ATRA, also responded in vitro to this agent with differentiation in granulocytes (Fig. 4B, panel $b$ ) and increased expression of RIZl gene (Fig. 3A). Expression of RIZl was unaffected by ATRA (Fig. 3A) or 1,25-dihydroxyvitamin $\mathrm{D}_{3}$ treatment (Fig. 3B) in the

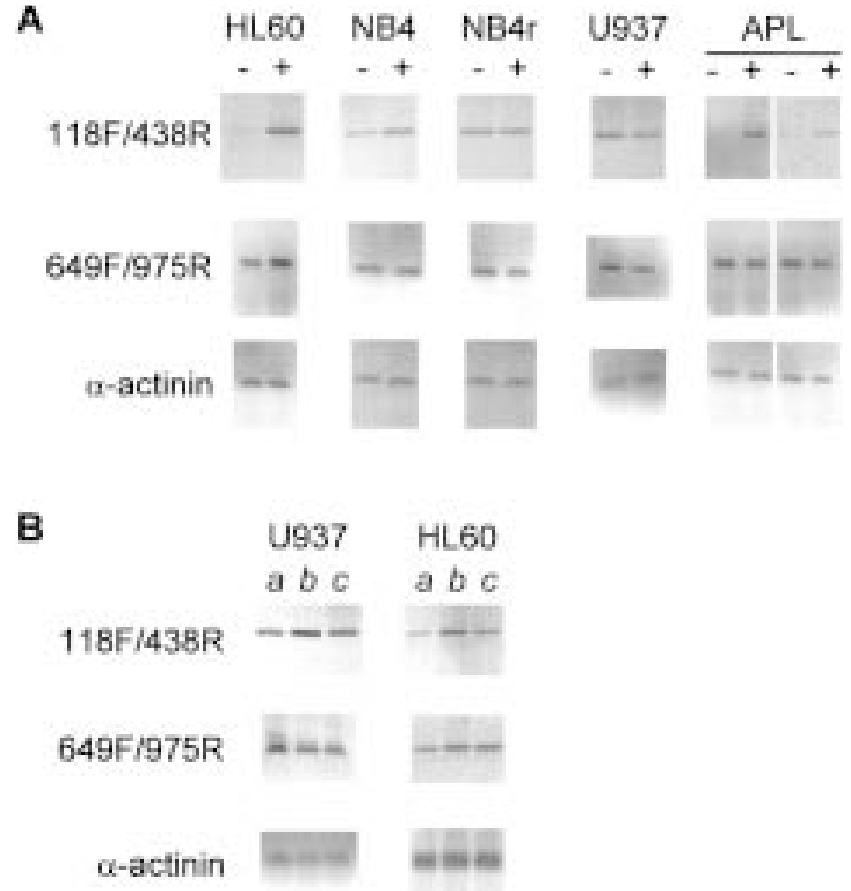

Fig. 3. Selective expression of RIZl induced by ATRA or other agents in different cell lines, or in leukemic patient promyelocytes. Electrophoretic analysis of the fragments obtained by RT-PCR of mRNA extracted from the indicated cell lines. On the left side is reported the set of primers used for amplification. (A) mRNA extracted from HL60, NB4, or NB4.007/6 (NB4r) cell lines or from leukemic patient promyelocytes (APL) untreated $(-)$ or treated with $1 \mu \mathrm{M}$ ATRA for 7 days $(+)$. (B) mRNA extracted from U937 or HL60 cell lines untreated $(a)$ or treated with $100 \mathrm{ng} / \mathrm{ml}$ of TPA $(b)$ or with $50 \mathrm{nM}$ 1,25-dihydroxyvitamin $\mathrm{D}_{3}(c)$ for 7 days.

human histiocytic lymphoma cell line, U937, that in our experimental conditions was not induced to differentiate by these agents. The same cell line, however, responded to TPA by differentiation in monocytes and a slightly increased expression of RIZ1 (Fig 3B). In HL60 cells, TPA (27) or 1,25dihydroxyvitamin $\mathrm{D}_{3}(28)$, that induced differentiation toward the macrophage-monocyte lineage, induced RIZl expression (Fig 3B).

Immunocytochemical staining of RIZ protein in HL60 cells indicated that ATRA treatment modified the intracellular distribution of the antigen. In untreated cells, staining with a monoclonal antibody to RIZ protein showed a faint cytoplasmic staining (Fig 4A, a). Nuclear staining appeared only after permeabilization of the fixed cells with Triton X-100 (Fig 4A, b). A 7-day treatment with ATRA produced the expected maturation of HL60 cells in granulocytes and immunocytochemical analysis showed a marked nuclear distribution of the antigen that was not affected by the previous permeabilization with the detergent (Fig. 4A, $c-d$ ). Nuclear distribution of another nuclear antigen, PCNA, was not modified by treatment with Triton X-100 (Fig. 4A, e-f). Immunocytochemical analysis of leukemic promyelocytes is 

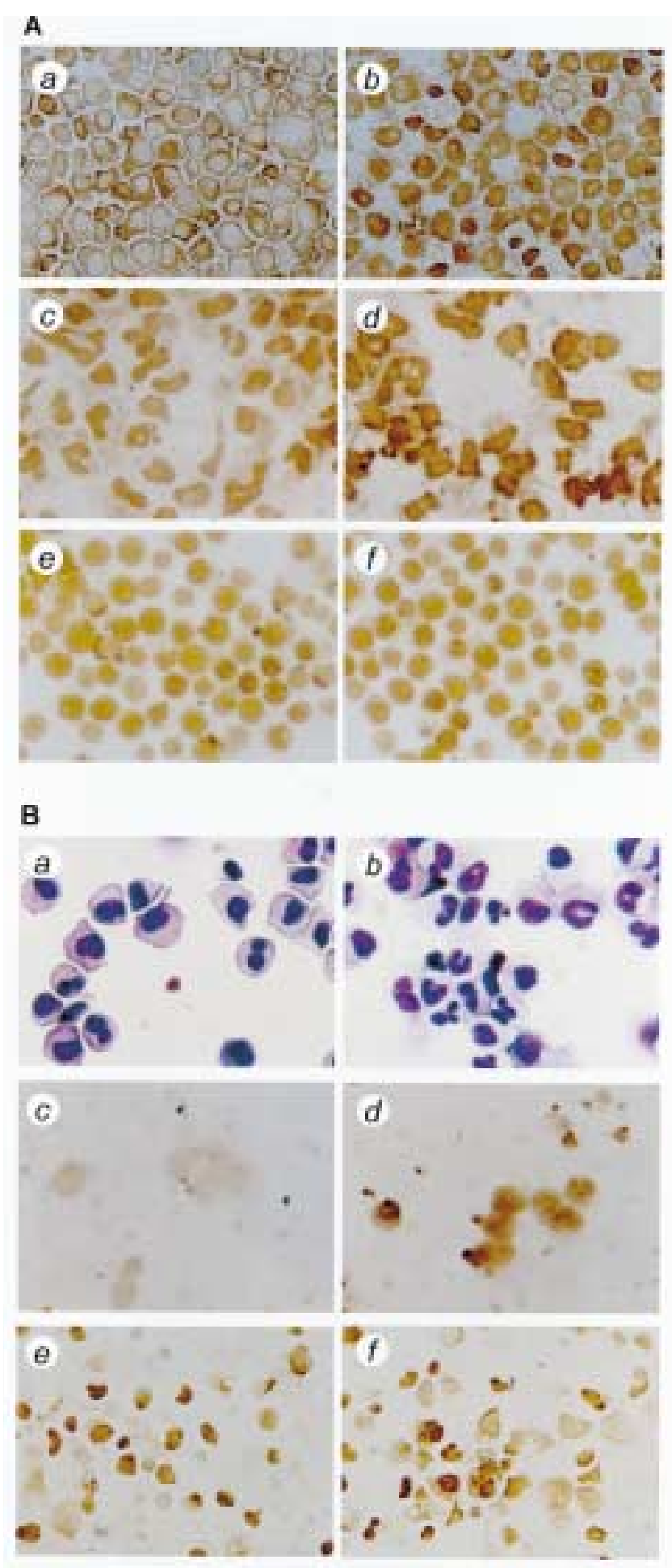

Fig. 4. Immunochemical analysis of RIZ protein. (A) HL60 cells were incubated in the presence (frames $c$ and $d$ ) or absence (frames $a-f$ ) of $1 \mu \mathrm{M}$ of ATRA. Frames $a-d$ : staining with antibody to RIZ protein RZ1723. Frames $e$ and $f$ : staining with antibody to PCNA. Frames $b, d$, and $f$ : permeabilization with Triton X-100 followed by staining with the specific antibody. (B) Mononucleated cells from acute promyelocytic leukemia patient were incubated in the presence (frames $b, e$, and $f$ ) or absence (frames $a, c$, and $d$ ) of $1 \mu \mathrm{M}$ of ATRA. Frames $a$ and $b$ : May-Grünwald staining. Frames $c, d, e$, and $f$ : immunoperoxidase staining with antibody to RIZ protein RZ1723. shown in panel B of Figure 4. Treatment with ATRA induced granulocytic differentiation of cultured cells, as evident from the May-Grünwald staining (Fig. 4B, $a-b$ ). The nuclear staining for RIZ protein was evident only after Triton X-100 treatment of fixed undifferentiated cells (Fig. 4B, $c-d$ ), whereas it was unaffected by the detergent in ATRA-differentiated cells (Fig. 4B, $e-f$ ).

To test whether the increased expression of RIZl was mediating the terminal differentiation of promyelocytes induced by ATRA or other agents, RIZ1 expression was forced in HL60 cells by infection with a recombinant adenovirus (AdRIZl) lacking the EIA and EIB regions and containing the rat RIZl cDNA (13). Infected cells showed no signs of differentiation but they died within 4 days (Fig. 5A). Acridine orange staining of infected cells showed a progressive permeabilization of the nucleus to the dye (Fig. 5B). Similar results were obtained by liposome-mediated transfection of patient leukemic promyelocytes with a large fragment of human RIZl cDNA inserted in an expression plasmid, pCT-RIZ(1-1245) (data not shown). The nuclear extracts from cells infected with AdRIZl were analyzed by Western blot with antibodies to RIZ protein (RZ1723) or to pl30 (SC-317). The results, presented in the panel $\mathrm{C}$ of Figure 5 , indicate that RIZ protein expression was induced by the adenovirus infection and that p130 expression was unaffected.

\section{Discussion}

Normal hematopoietic cells follow a fixed developmental sequence and can undergo cell division only in early stages of maturation. Differentiation in the mature phenotype is associated with arrest of cell division and acquisition of final features. The transition from the proliferative to the maturational phase is regulated by hematopoietic growth factors. In leukemic cells, this transition does not occur and, therefore, not only cells remain indefinitely capable of cell division, but they also suppress the apoptotic program that is the normal epilogue of their life cycle. Some anti-leukemic agents act to induce apoptosis; others, such as ATRA in promyelocytic leukemia, restore the transition to the mature phenotype. HL60 is a widely used cell line derived from promyelocytic leukemia. Cell treatment with ATRA or 1,25-dihydroxyvitamin $\mathrm{D}_{3}$ produces growth arrest and differentiation in granulocyte- or monocyte-like cells, respectively. We used this model to investigate the molecular mechanisms involved in this transition, examining the behavior of RIZ protein. This protein is described as negative regulator of cell growth and tumorigenesis because, like other PR domain-containing gene products, it is underexpressed in neoplastic tissue or expressed in a PRdefective form (13). We present here experimental evidence that in HL60 cell line and in promyelocytes 
a

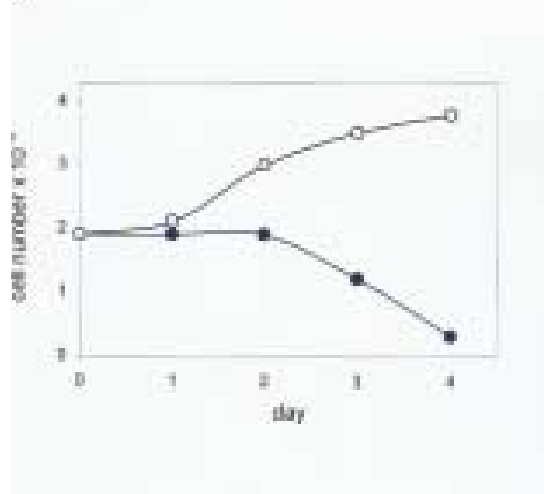

日

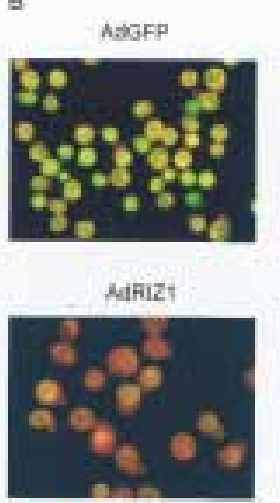

c

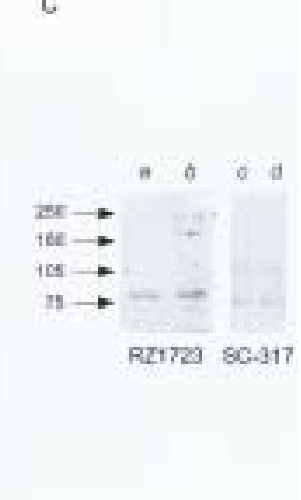

Fig. 5. Growth inhibition by forced RIZ1 expression. (A) growth curve of HL60 infected with the adenovirus vector AdRIZl $(\bullet)$ or AdGFP $(\bigcirc)$. Infected cells were plated in $60-\mathrm{mm}$ dishes and counted at the indicated times. (B) Fluorescence microphotographs of acridine orangestained HL60 cells after $72 \mathrm{hr}$ from transfection with the adenovirus vector AdRIZl or AdGFP, as indicated. (C) Western blot analysis of nuclear extract of HL60 cells infected with the adenovirus vector AdGFP (lanes $a$ and $c$ ) or AdRIZ1 (lanes $b$ and $d$ ) with the antibody RZ1723 to RIZ protein or SC-317 to p130, as indicated below. Arrows on the sides indicate the migration of standard proteins $(\mathrm{kDa})$. of ATRA-sensitive APL, RIZ1 expression was selectively modulated during the differentiative response induced by retinoic acid and it was maximal at 7 days, when all cells displayed a differentiated phenotype. In HL60 cells, retinoic acid also induced the expression of p130, the retinoblastoma tumor suppressor homolog known for blocking hematopoietic cell proliferation, but not of $\mathrm{pRb}$. Differentiation was also associated with a modification of the immunochemical nuclear staining of RIZ protein. Furthermore, in HL60 cells, stimulation of an alternative maturation pathway, based on rexinoid and protein kinase A crosstalk (25), produced the same effect on RIZl induction. In all tested myeloid cell lines, agents inducing differentiation selectively increased the expression of the complete, PR-containing RIZl form. TPA, in fact, was ineffective in HL60 cells, but increased RIZl expression in U937 cells, where it induced monocytic differentiation. In the NB4 lines, ATRA effect on RIZl expression was evident only in the sensitive but not in the resistant cells and correlated with the differentiation. In synthesis, the selective increase of RIZl transcript was induced by the tested agents only in those cells in which they promoted differentiation. These results suggest that RIZ1 expression protein is a common effect of agents promoting myeloid cell differentiation and the evidence that RIZl expression is increased by the activation of different signaling pathways indicates that RIZl gene product act downstream of those pathways. The selective RIZl expression correlates to myeloid maturation, while giving further support to the postulated yin-yang role of PR-containing proteins in proliferation control. It also suggests that RIZ protein could be involved in the proliferation/ differentiation transition.

In HL60 cells, as well as in leukemic promyelocytes, immunolocalization showed that RIZ protein was present in both cytoplasm and nucleus. However, in the nucleus it was accessible to the antibody only after a short application of a Triton X-100 solution to the fixed cells. In ATRA-differentiated cells, nuclear localization was revealed without detergent treatment. Although we have no clue on the nature of this epitope concealment in the undifferentiated phenotype, it is important to consider that it occurred also in MCF-7 cells when stimulated by estradiol to proliferate (15). We therefore interpret this phenomenon as an intranuclear confinement of RIZ protein and/or of its PR domain. Removal of an active PR domain from the nucleus of neoplastic cells appears to be a common feature of many PR domain-containing proteins. In tumor cells, this is achieved in various ways by the different genes of the family (e.g., chromosome translocation, point mutation, chromosome deletion). It is therefore conceivable that the observed phenomenon of epitope concealment in actively proliferating cells is the epigenetic equivalent for PR domain inactivation. Alternatively, epitope masking could be consequent to participation of RIZ protein in complex functional aggregates with other proteins (e.g., pRb) to remove growth inhibition. Differentiating agents capable of arresting growth (and eventually promoting apoptosis) could revert this phenomenon. In our experimental model, both intranuclear redistribution of the RIZ gene product and de novo synthesis of RIZl restores active intranuclear PR domains. For another gene of the family, the same effect was achieved by induction of nuclear translocation upon serum starvation or nerve growth factor treatment (11).

Forced expression of RIZ1 protein was lethal for HL60 cells. We have no evidence as to whether this phenomenon was mediated by death receptor activation or by mitochondrial permeability. However, this finding is consistent with the cell growth arrest and apoptosis observed in breast cancer and osteosarcoma cell lines upon infection with the same adenovirus vector (13). Transfection of cell lines with other genes of the PR-containing protein family produces similar results in other systems. Blimp-1, a protein involved in the maturation of B-lymphocytes, causes apoptosis in Abelson-transformed precursor B lymphocytes or differentiation in B-lymphoma cell lines (29). The forced expression of different forms of MDS1/EVI1 
gene derived by gene truncation or gene fusion (EVIl or AML1/MDS1/ EVIl) affects the TGF $\beta 1$ signaling pathway and G-CSF-induced differentiation of hematopoietic precursor cell line $32 \mathrm{Dcl} 3$ (30). It is, therefore, conceivable that the PR domain-containing family of protein is involved in the control of differentiation in response to extracellular stimuli. To complete the differentiation program, the cell requires the activation of converging pathways (e.g., c-myc downregulation, $\mathrm{pRb}$ expression) leading to the intranuclear accumulation of molecules directing the expression of differentiation genes, when properly assembled, or activating the apoptotic program. It is, therefore, not paradoxical that, despite the fact that PR domaincontaining genes are considered antioncogenes, their forced expression in certain conditions promotes cell death. It is important to consider that, in HL60 cells, forced expression of RIZ protein did not modify p130 expression.

In conclusion, our presented evidence suggested that RIZ gene product could be a regulator of the proliferation/differentiation transition in myeloid cells and therefore it could become a candidate target for selective cancer therapy. More extensive investigations in other models (e.g., lymphocytes, hematopoietic stem cells) could confirm a general role of RIZ gene products in the transition from proliferative activity to a quiescent/differentiate state and vice versa.

\section{Acknowledgments}

We thank Dr. She Huang (The Burnham Institute, La Jolla, CA, USA) for providing the AdRIZl and AdGFP adenovirus vectors, Dr. Mirco Fanelli (Istituto Europeo di Oncologia, Milano, Italy) for the NB4.007/6 cell line, and Dr. Saverio Minucci (Istituto Europeo di Oncologia, Milano, Italy) for helpful discussions.

This investigation was supported by the Italian Ministry for University and Scientific and Technological Research.

\section{References}

1. Buyse IM, Shao G, Huang S. (1995) The retinoblastoma protein binds to RIZ, a zinc-finger protein that shares an epitope with the adenovirus ElA protein. Proc. Natl. Acad. Sci. U.S.A. 92: 4467-4471.

2. Muraosa Y, Takahashi K, Yoshizawa M, Shibahara S. (1996) cDNA cloning of a novel protein containing two zinc-finger domains that may function as a transcription factor for the human heme-oxygenase-1 gene. Eur. J. Biochem. 235: 471479.

3. Xie M, Shao G, Buyse I, Huang S. (1997) Transcriptional repression mediated by the PR domain zinc finger gene RIZ. J. Biol. Chem. 272: 26360-26366.

4. Shapiro VS, Lee P, Winoto A. (1995) Identification and cloning of the G3B cDNA encoding a 3' segment of a protein binding to GATA-3. Gene 163: 329-330.

5. Buyse IM, Takahashi EI, Huang S. (1996) Physical mapping of the retinoblastoma interacting zinc finger gene RIZ to D1S228 on chromosome 1p36. Genomics 34: 119121 .
6. Chadwick RB, Jiang GL, Bennington GA, et al. (2000) Candidate tumor suppressor RIZ is frequently involved in colorectal carcinogenesis. Proc. Natl. Acad. Sci. U.S.A. 97: 2662-2667.

7. Liu L, Shao G, Steele-Perkins G, Huang S. (1997) The retinoblastoma interacting zinc finger gene RIZ produces a PR domain-lacking product through an internal promoter. J. Biol. Chem. 272: 2984-2991.

8. Fears S, Vignon C, Bohlander SK, Smith S, Rowley JD, Nucifora G. (1996) Intergenic splicing of MDS I and EVIl occurs in normal tissues as well as in myeloid leukemia and produces a new member of the PR domain family. Proc. Natl. Acad. Sci. U.S.A. 93: 1642-1647.

9. Turner CAJ, Mack DH, Davis MM. (1994) Blimp-1, a novel zinc finger-containing protein that can drive the maturation of B lymphocytes into immunoglobulin-secreting cells. Cell 77: 297-306.

10. Messika EJ, Lu PS, Sung YJ, et al. (1998) Differential effect of B lymphocyte-induced maturation protein (Blimp-1) expression on cell fate during B cell development. J. Exp. Med. 188 515-525.

11. Chittka A, Chao MV. (1999) Identification of a zinc finger protein whose subcellular distribution is regulated by serum and nerve growth factor. Proc. Natl. Acad. Sci. U.S.A. 96: 1070510710.

12. Yang X, Huang S. (1999) PFMl (PRDM4), a new member of the PR-domain family, maps to a tumor suppressor locus on human chromosome 12q23-q24.1. Genomics 61: 319-325.

13. He L, Yu JX, Liu L, et al. (1998) RIZ1, but not the alternative RIZ2 product of the same gene, is underexpressed in breast cancer, and forced RIZl expression causes G2-M cell cycle arrest and/or apoptosis. Cancer Res. 58: 4238-4244.

14. Jiang GL, Liu L, Buyse IM, Simon D, Huang S. (1999) Decreased RIZ1 expression but not RIZ2 in hepatoma and suppression of hepatoma tumorigenicity by RIZ1. Int. J. Cancer 83 : 541-546.

15. Abbondanza C, Medici N, Nigro V, et al. (2000) The retinoblastoma-interacting zinc-finger protein RIZ is a downstream effector of estrogen action. Proc. Natl. Acad. Sci. U.S.A. 97: 3130-3135.

16. Grignani F, Fagioli M, Alcalay M, et al. (1994) Acute promyelocytic leukemia: from genetics to treatment. Blood 83: $10-25$.

17. Slack JL, Gallagher RE. (1999) The molecular biology of acute promyelocytic leukemia. Cancer Treat. Res. 99: 75-124.

18. Breitman TR, Collins SJ, Keene BR. (1981) Terminal differentiation of human promyelocytic leukemic cells in primary culture in response to retinoic acid. Blood 57: 10001004.

19. Lo Coco F, Diverio D, Falini B, Biondi A, Nervi C, Pelicci PG. (1999) Genetic diagnosis and molecular monitoring in the management of acute promyelocytic leukemia. Blood 94: 12-22.

20. Fanelli M, Minucci S, Gelmetti V, Nervi C, GambacortiPasserini C, Pelicci PG. (1999) Constitutive degradation of PML/RARalpha through the proteasome pathway mediates retinoic acid resistance. Blood 93: 1477-1481.

21. Medici N, Abbondanza C, Nigro V, et al. (1999) Identification of a DNA binding protein cooperating with estrogen receptor as RIZ (retinoblastoma interacting zinc finger protein). Biochem. Biophys. Res. Commun. 264: 983-989.

22. Lee WS, Kao CC, Bryant GO, Liu X, Berk AJ. (1991) Adenovirus ElA activation domain binds the basic repeat in the TATA box transcription factor. Cell 67: 365-376.

23. Dignam JD, Lebovitz RM, Roeder RG. (1983) Accurate transcription initiation by RNA polymerase II in a soluble extract from isolated mammalian nuclei. Nucleic Acids Res. 11: 1475-1489.

24. Breitman T, Selonick S, Collins S. (1980) Induction of differentiation of the human promyelocytic leukemia cell line (HL60) by retinoic acid. Proc. Natl. Acad. Sci. U.S.A. 77: 2936-2940.

25. Benoit G, Altucci L, Flexor M, et al. (1999) RAR-independent RXR signaling induces $t(15 ; 17)$ leukemia cell maturation. ЕМВО J. 18: 7011-7018. 
26. Mori A, Higashi H, Hoshikawa Y, Imamura M, Asaka M Hatakeyama M. (1999) Granulocytic differentiation of myeloid progenitor cells by p130, the retinoblastoma tumor suppressor homologue. Oncogene 18: 6209-6221.

27. Koeffler HP, Bar-Eli M, Territo MC. (1981) Phorbol ester effect on differentiation of human myeloid leukemia cell lines blocked at different stages of maturation. Cancer Res. 41: 919-926.

28. McCarthy DM, San Miguel JF, Freake HC, Green PM, Zola H, Catovsky D, Goldman JM. (1983) 1,25-dihydroxyvitamin D3 inhibits proliferation of human promyelocytic leukaemia (HL60) cells and induces monocyte-macrophage differentiation in HL60 and normal human bone marrow cells. Leuk. Res. 7: 51-55.

29. Lin Y, Wong K, Calame K. (1997) Repression of c-myc transcription by Blimp-1, an inducer of terminal B cell differentiation. Science 276: 596-599.

30. Sood R, Talwar-Trikha A, Chakrabarti SR, Nucifora G. (1999) MDS1/EVIl enhances TGF-betal signaling and strengthens its growth-inhibitory effect but the leukemia-associated fusion protein AML1/MDS1/EVI1, product of the $t(3 ; 21)$, abrogates growth-inhibition in response to TGF-betal. Leukemia 13: $348-357$. 